\title{
Comparative study of the best achievable contrast in scalar, Stokes and Mueller images.
}

\author{
F. Goudail ${ }^{1}$, A. Bénière ${ }^{1,2}$, M. Alouini ${ }^{3}$, D. Dolfi ${ }^{2}$ \\ ${ }^{1}$ Laboratoire Charles Fabry de l'Institut d'Optique, CNRS, Univ Paris-Sud, RD 128, 91127 Palaiseau, France. \\ ${ }^{2}$ Thales research and Technology - France, RD128, 91767 Palaiseau. \\ ${ }^{3}$ Institut de physique de Rennes, CNRS, Univ Rennes 1, 35042 Rennes, France.
}

\begin{abstract}
We compare the relative performance of different active polarimetric imaging architectures for target detection applications. We show that if the noise that affects the measurements is additive and if the only relevant parameter is the contrast between an object of interest and a background with different Mueller matrices, the most efficient imaging architecture consists in acquiring a single intensity image while optimizing the illumination and analysis states of polarization.
\end{abstract}

\section{Introduction}

Polarization imaging consists in forming an image of the polarization state of the light backscattered by each point of a scene. It can reveal contrasts that do not appear in classical intensity images or provide information about the nature of the objects present in the scene (surface state, orientation). It has many applications in remote sensing [1], imaging through turbid media [2], biomedical imaging [3], industrial control [4].

There exist different types of polarimetric imaging systems. Passive ones rely on natural light sources (reflection of sunlight or emission) whereas active ones illuminate the scene with an artificial light source. In all cases, the light coming from the scene is analyzed by polarization modulators before forming intensity images. Four intensity images are necessary to estimate the full polarization state of the light (Stokes vector) and sixteen to characterize the intrinsic properties of the scene with respect to polarization (Mueller matrix). Cost and technological complexity of polarimetric imagers depend on the number of parameters they measure.

A key issue in the design of a polarimetric imaging system is thus to evaluate the added value of each measured polarimetric parameter in order to optimize the compromise between complexity and efficiency. In target detection applications, the relevant efficiency criterion is the contrast between a target region $a$ and a background region $b$ (see Figure 1). We will assume that the data acquisition is only perturbed by additive Gaussian noise. Our purpose will be to determine the best achievable contrast in three different polarization imaging modalities: scalar, Stokes and Mueller.

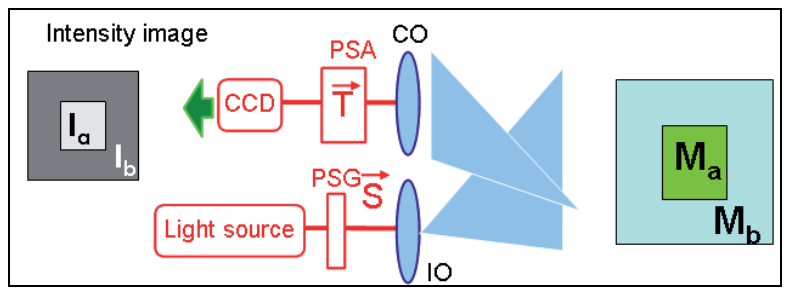

Figure 1 : Principle of active polarimetric imaging. PSG : Polarization State Generator. PSA : Polarization State Analyzer. IO : Illumination Optics. CO : collection optics.

We consider that the target region has a Mueller matrix $\mathrm{M}_{\mathrm{a}}$ and the background region a Mueller matrix $\mathrm{M}_{\mathrm{b}}$. The scene is illuminated with purely polarized light that can have any Stokes vector $\vec{S}$ on the Poincaré sphere and is produced by a Polarization State Generator (PSG) (see Figure 1). The Stokes vector of the light scattered by the region $a$ (b) is $\vec{S}_{a}=M_{a} \vec{S}\left(\vec{S}_{b}=M_{b} \vec{S}\right)$. The light scattered by the scene is analyzed by a Polarization State Analyzer (PSA) whose eigenstate is the unit intensity, purely polarized Stokes vector $\vec{T}$. In the following, we will consider three different types of polarimetric imaging architectures: 
- Scalar imaging, where only one intensity image is acquired.

- Stokes imaging, where the whole Stokes vector is measured (4 different illumination states are used, 4 intensity images are acquired).

- $\quad$ Mueller imaging, where the whole Mueller matrix is measured (4 illumination states and 4 analysis states are used, 16 intensity images are acquired).

In this paper, we will determine the maximal target/background contrast that can be achieved by these three architectures. This will make it possible to compare them on an objective basis and to choose the one that is most adapted to target detection applications.

\section{Contrast in scalar imaging}

Let us first consider the scalar imaging configuration. At a given pixel of regions $a$ or $b$, the measured intensity is [5]:

$$
\begin{aligned}
& I_{a}=t_{\mathrm{int}} \times \frac{1}{2} \vec{T}^{T} M_{a} \vec{S}+n_{a} \\
& I_{b}=t_{\mathrm{int}} \times \frac{1}{2} \vec{T}^{T} M_{b} \vec{S}+n_{b}
\end{aligned}
$$

In this equation, $\vec{T}$ and $\vec{S}$ are unit intensity Stokes vectors, $t_{i n t}$ is the integration time and $n_{a}, n_{b}$ are zero mean Gaussian random variables with standard deviation $\sigma$. It has to be noted that the standard deviation $\sigma$ of the noise may depend on $t_{i n t}$. Indeed, it is independent of $t_{\text {int }}$ in the case of readout noise and proportional to $\sqrt{t_{\text {int }}}$ in case of dark current noise or shot noise due to background illumination. We shall see that this difference has a significant impact on the value of the achievable contrast.

The expression of the contrast between a target and a background in the additive Gaussian noise model is [6]:

$$
C(\vec{S}, \vec{T})=\frac{\left(I_{a}-I_{b}\right)^{2}}{\sigma^{2}}
$$

Using Eq. 1, this contrast can also be written as

$$
C(\vec{S}, \vec{T})=\left(\frac{t_{\mathrm{int}}}{\sigma}\right)^{2} \times \frac{1}{4}\left(\vec{T}^{T} \Delta M \vec{S}\right)^{2}
$$

where

$$
\Delta M=M_{a}-M_{b}
$$

is the difference between the Mueller matrices of the two regions. This contrast is a function of both the illumination and the analysis states. In practice, one will determine the vectors $\vec{S}$ and $\vec{T}$ that maximize it. Finally, the maximal achievable contrast is [5]:

$$
C_{\text {scal }}=\max _{\vec{S}, \vec{T}}[C(\vec{S}, \vec{T})]
$$

\section{Contrast in Stokes imaging}

Let us now consider Stokes imaging systems, where 4 different analysis polarization states are used. Let us define the matrix $W$ whose lines are the Stokes vectors of the four analysis states. These four states of polarization are usually chosen so as to minimize the variance of the estimated Stokes vector $[7,8]$. The 4 measured intensities can be stacked in fours dimensional vectors:

$$
\begin{aligned}
& \vec{I}_{a}=\frac{t_{\text {int }}}{4} \times \frac{1}{2} W M_{a} \vec{S}+\vec{n}_{a} \\
& \vec{I}_{b}=\frac{t_{\text {int }}}{4} \times \frac{1}{2} W M_{b} \vec{S}+\vec{n}_{b}
\end{aligned}
$$

where the vectors $\vec{n}_{u}, u=a, b$, are 4-dimensional white Gaussian random vectors with standard deviation $\sigma$. The factor $t_{\text {int }} / 4$ comes from the fact that the measurement time is constant, so that each of the four intensity measurements is done during one quarter of the available time. The expression of the contrast is [9] :

$$
C(\vec{S})=\left(\frac{t_{\text {int }}}{4 \sigma}\right)^{2} \times \frac{1}{4}\left(\vec{S}^{T} G \vec{S}\right)^{2}
$$

where

$$
G=\Delta M^{T} W^{T} W \Delta M
$$

It is a function of the illumination state. In practice, one will determine the vector $\vec{S}$ that maximizes it, and the maximal achievable contrast is [9] :

$$
C_{\text {stok }}=\max _{\vec{S}}[C(\vec{S})]
$$

\section{Contrast in Mueller imaging}

Let us now consider Mueller imaging systems. In this case, one uses four different illumination Stokes vectors that are in general identical to the analysis vectors used for Stokes imaging. One also has four analysis states and one thus measures 16 intensities that can be gathered in the following matrices:

$$
\begin{aligned}
& I_{a}=\frac{t_{\mathrm{int}}}{16} \times W M_{a} W^{T}+N_{a} \\
& I_{b}=\frac{t_{\mathrm{int}}}{16} \times \frac{1}{2} W M_{b} W^{T}+N_{b}
\end{aligned}
$$


where the matrix $W$ has been defined in the previous section. The matrices $N_{a}$ and $N_{b}$ are $4 \times 4$ random matrix whose elements are independent Gaussian random values with standard deviation $\sigma$ and the factor $t_{\text {int }} / 16$ stands for the fact that 16 intensity measurements are performed during the time interval $t_{\text {int }}$. The contrast can be written as:

$$
C_{\text {muel }}=\left(\frac{t_{\text {int }}}{16 \sigma}\right)^{2} \times \frac{1}{4}\left\|W \Delta M W^{T}\right\|^{2}
$$

where $\| \cdots \cdot$ denotes the Frobenius norm. It does not depend on any free parameter.

\section{Comparison of the different architectures}

It is interesting to compare the values of the contrast that that can be reached by these three different architectures in a given application. For that purpose, let us consider the following scenario. We consider that the target has the following Mueller matrix:

$$
M_{\alpha}=\left[\begin{array}{cccc}
1 & 0 & 0 & \gamma \\
0 & 0.5 & 0 & 0 \\
4 \gamma & 0 & 0.3 & 0 \\
-\gamma & 0 & 0 & 0.4
\end{array}\right]
$$

where $\gamma$ is a scalar parameter that can vary from 0 to 0.1 . We have checked that for all these values of $\gamma$, the matrix $M_{a}$ remains physical. We also assume that the background region has the following diagonal Mueller matrix :

$$
M_{b}=\left[\begin{array}{cccc}
0.8 & 0 & 0 & 0 \\
0 & 0.3 & 0 & 0 \\
0 & 0 & 0.3 & 0 \\
0 & 0 & 0 & 0.4
\end{array}\right]
$$

We have plotted in Figure 2 the square roots of the values of the contrasts $C_{\text {scal }}, C_{\text {stok }}, C_{\text {muel }}$ obtained with the three architectures as the parameter $\gamma$ varies. For the Stokes and Mueller architectures, there are two curves. The solid curves correspond to the case where the noise variance $\sigma^{2}$ is independent of the measurement time $t_{\text {int }}$, such as in the case of readout noise. In this case, it is easily seen in Eqs 3, 7 and 11 that the contrast decreases proportionally to the number of measurements. This is easily understood since each measurement adds up a constant amount of noise to the measurement, thus reducing the contrast. The dotted curves correspond to the case of dark current noise or background photon noise, where the additive noise variance is proportional to $t_{\text {int }}$ and can be written $\sigma^{2}=a t_{\text {int }} / N$, where $N$ is the number of measurements and $a$ is a positive real-valued number.

It is clearly seen that for all values of $\gamma$, the maximal contrast is obtained with the scalar imaging architecture. Then comes the Stokes architecture and finally the Mueller one. In conclusion, to maximize the contrast, it is preferable to make as few measurement as possible, as soon as these measurements performed with optimized illumination and analysis polarization states.

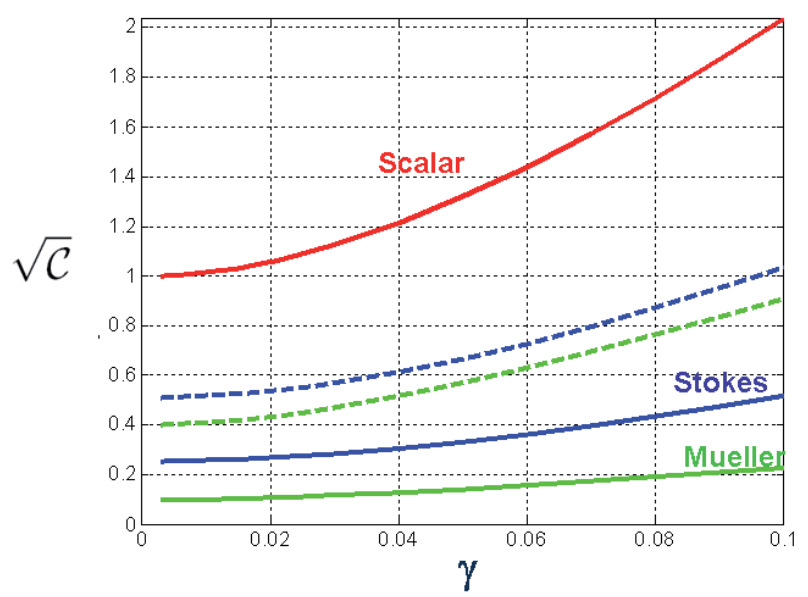

Figure 2 : Variation of the square roots of the contrasts $C_{\text {scal }}, C_{\text {stok }}, C_{\text {muel }}$ as a function of $\gamma$. Solid lines : noise variance is constant. Dotted lines : noise variance is proportional to $t_{\text {int }}$.

In order to have an idea of the gain in contrast obtained by using the scalar imaging architecture, we have plotted the following ratios

$$
\rho=\sqrt{\frac{\mathcal{C}_{\text {Scalar }}}{\mathcal{C}_{\text {Stokes }}}} \text { or } \sqrt{\frac{\mathcal{C}_{\text {Scalar }}}{\mathcal{C}_{\text {Mueller }}}}
$$

as a function of $\gamma$. It is seen that when the noise variance is independent of $t_{\text {int }}$, the gain is of a factor 10 compared to Mueller architecture and of 4 with respect to Stokes imagers. When the noise variance is proportional to $t_{\text {int }}$, the gain factor is more modest but still around 2 compared to Mueller and Stokes imaging. 


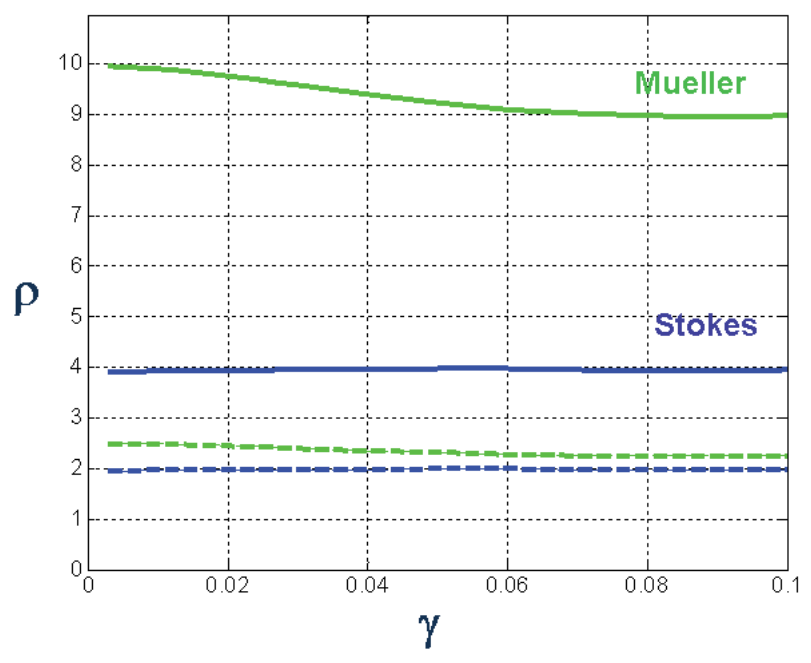

Figure 3 : Variation of the contrast ratio $\rho$ as a function of $\gamma$. Solid lines : noise variance is constant. Dotted lines : noise variance is proportional to $t_{i n t}$.

\section{Conclusion}

In conclusion, it can be said that for the considered target detection application, scalar imaging is the most efficient approach and that all architectures acquiring more than two images will lead to a lower contrast.

Of course, this conclusion is valid only if the only concern is the target/background contrast. For other applications, where it is necessary to have deeper physical insight in the physics of the target and/or the background, Stokes and Mueller imaging may be preferable. It may also be the case when more than two regions have to be discriminated.

This work has many perspectives. For example, it will be interesting to consider other noise models than the simple additive noise, such as signal dependent photon shot noise [10], speckle noise [11], or variability of the polarimetric properties of the scene.

\section{References}

1. S. Breugnot, P. Clémenceau,, Opt. Eng. 39, 26812688 (2000).

2. R.E. Nothdurft and G. Yao, Appl. Opt. 45, 55325541 (2006).

3. S. L. Jacques, J. C. Ramella-Roman, K. Lee, J. Biomed. Opt. 7, 329-340 (2002).

4. O. Morel, C. Stolz, F. Meriaudeau, P. Gorria, App. Opt. 45, 4062-4068 (2006).

5. F. Goudail, Opt. Lett. 34, 121-123 (2009).

6. F.Goudail, P. Réfrégier, G. Delyon, J. Opt. Soc. Am. A 21, 1231-1240 (2004).

7. A. Ambirajan, D. C. Look, Opt. Eng. 34, 1651-1658 (1995).

8. J. S. Tyo, Opt. Lett. 25, 1198-1200 (2000).
9. F. Goudail, A. Bénière, Opt. Lett. 34, 1471-1473 (2009).

10. F. Goudail, Opt. Lett. 34, 647-649 (2009).

11. F. Goudail, P. Réfrégier, J. Opt. Soc. Am. A 18, 3049-3060 (2001). 